Journal of Fluid Mechanics

http://journals.cambridge.org/FLM

Journal of

Additional services for Journal of Fluid Mechanics:

Email alerts: $\underline{\text { Click here }}$

Subscriptions: $\underline{\text { Click here }}$

Commercial reprints: $\underline{\text { Click here }}$

Terms of use : $\underline{\text { Click here }}$

\title{
The disappearance of laminar and turbulent wakes in complex flows
}

\author{
J. C. R. HUNT and I. EAMES
}

Journal of Fluid Mechanics / Volume 457 / April 2002, pp 111 - 132

DOI: 10.1017/S0022112001007236, Published online: 09 April 2002

Link to this article: http://journals.cambridge.org/abstract_S0022112001007236

How to cite this article:

J. C. R. HUNT and I. EAMES (2002). The disappearance of laminar and turbulent wakes in complex flows. Journal of Fluid Mechanics, 457, pp 111-132 doi:10.1017/S0022112001007236

Request Permissions : $\underline{\text { Click here }}$ 


\title{
The disappearance of laminar and turbulent wakes in complex flows
}

\author{
By J. C. R. HUNT $T^{1} \dagger$ AND I. EAMES $\mathbf{S}^{2}$ \\ ${ }^{1}$ Departments of Space and Climate Physics and Geological Sciences, University College London, \\ Gower Street, London WC1E 6BT, UK \\ ${ }^{2}$ Department of Mechanical Engineering, University College London, Gower Street, \\ London WC1E 7JE, UK
}

(Received 7 June 2001 and in revised form 18 September 2001)

The singular effects of steady large-scale external strain on the viscous wake generated by a rigid body and the overall flow field are analysed. In an accelerating flow strained at a positive rate, the vorticity field is annihilated owing to positive and negative vorticity either side of the wake centreline diffusing into one another and the volume flux in the wake decreases with downwind distance. Since the wake disappears, the far-field flow changes from monopolar to dipolar. In this case, the force on the body is no longer proportional to the strength of the monopole, but is proportional to the strength of the far field dipole. These results are extended to the case of strained turbulent wakes and this is verified against experimental wind tunnel measurements of Keffer (1965) and Elliott \& Townsend (1981) for positive and negative strains. The analysis demonstrates why the total force acting on a body may be estimated by adding the viscous drag and inviscid force due to the irrotational straining field.

Applying the analysis to the wake region of a rigid body or a bubble shows that the wake volume flux decreases even in uniform flows owing to the local straining flow in the near-wake region. While the wake volume flux decreases by a small amount for the flow over streamline and bluff bodies, for the case of a clean bubble the decrease is so large as to render Betz's (1925) drag formula invalid.

To show how these results may be applied to complex flows, the effects of a sequence of positive and negative strains on the wake are considered. The average wake width is much larger than in the absence of a strain field and this leads to diffusion of vorticity between wakes and the cancellation of vorticity. The latter mechanism leads to a net reduction in the volume flux deficit downstream which explains why in calculations of the flow through groups of moving or stationary bodies the wakes of upstream bodies may be ignored even though their drag and lift forces have a significant effect on the overall flow field.

\section{Introduction}

When a flow is forced through groups of moving or stationary bodies, such as bubbles, spray droplets, boiler tubes, or blade-rows of turbines, measurements and computations show that both the local acceleration and deceleration of the flow and the vortical wakes from upwind affect the forces acting on these bodies. However, a common assumption, based on some measurements, is that if the bodies are quite

$\dagger$ Also at: J. M. Burgers Centre, Delft University of Technology. 
closely packed these wakes partially or completely 'disappear' in the distorted flow around the obstacles. The same phenomenon occurs in the velocity fields between groups of vortices because they also shed vorticity into their wakes. Because of this effect, in local calculations of close-packed multibody (and multivortex) flows, any significant perturbation caused by the wakes of upwind bodies may, to the first approximation be neglected (Hunt, Perkins \& Fung 1994; Rollet-Miet, Laurence \& Ferziger 1999). Since the drag on a bluff body (or a vortex) is related to the momentum defect of its wake (Betz 1925), how this force is related to the far-field flow around the body when the wake 'disappears' needs explaining. This relates to the convenient approximation of neglecting the wakes of bodies far upwind. If the spacing between the bodies is much greater than their diameters, the wakes may be slightly affected by interactions, but the vorticity they generate significantly influences the flow produced far from each body. Although this is ignored in idealized two-phase flow models, its effect on the bulk properties of two-phase flows is appreciable (Hunt et al. 1994).

The physical reason for this widespread tendency of wakes to disappear is that in accelerating flows, the opposite signed vorticity on each side of the wake, that normally diffuses outwards, is confined and distorted by the mean flow. Then it diffuses across the wake and is cancelled out. By contrast, in two-dimensional diverging flows, cross-stream diffusion is reduced and the vorticity magnitude tends to a constant value, so a different argument is needed to justify the neglect of wakes of upwind obstacles. We show here that the interaction between wide wakes also leads to vorticity cancellation and their net effect is eliminated downstream. Although the effect of converging/diverging streamlines on an external turbulent wake was analysed and measured by Keffer (1965) and Elliott \& Townsend (1981), the wider implications for computing complex flows were not explored. In particular, Keffer did not consider how the disappearance of the wake affected the external field and how it could be consistent with the drag force on the body causing the wake, since the drag on the body is normally found to be proportional to the wake strength. A related question is how a sequence of converging and diverging flows affects a wake; does it simply diffuse, acting like an enhanced turbulent diffusivity or can the external straining flow destroy the wake by enhanced vorticity cancellation?

The phenomenon of vorticity cancelling always occurs to some extent in the near wake of the viscous flow around any shape of body, even without any external straining flow. This cancellation, which is associated with a strong local acceleration and a convergence of the mean streamlines in the near-wake region, also produces a dipole perturbation in the external flow. This transition, which has been analyzed in the wake of a flat plate (e.g. Talke \& Berger 1970), shows how a strong coupling between the near-field wake and the boundary-layer flow over a flat plate amplifies the skin friction near the trailing edge of aircraft. Mathematically, this becomes an eigenvalue problem with the flow adjusting over a characteristic length. The latter effect influences the forces acting on the body when it is placed in a shear (Bowles \& Smith 2000) or accelerating flow. Partial vorticity cancellation is demonstrated to be the key mechanism relating the strength of the far-field wake to the separated flow over the body or to the vorticity flux in unseparated boundary layers, such as those on bubbles (in clean water) (Moore 1963). In some accelerating and unsteady flows, this phenomenon of wake disappearance may explain why, as we shall show, the forces on the body can be calculated using inviscid theory for drag forces on bodies in accelerating flows, but allowing for the effect of the viscous wake near the body on the inviscid flow (Magnaudet \& Eames 2000). 
The aims of this paper are (i) to derive solutions for the mean velocity in planar and axisymmetric wakes in straining flows for both laminar and turbulent flows, (ii) to relate these to the perturbed flow field around the whole body and to the force acting on the bodies, and (iii) to consider wakes in complex straining flows with positive and negative strains. Thence we draw some general conclusions.

\section{Linear effects of uniform strain on a laminar wake}

The effect of an irrotational straining external flow, $\boldsymbol{U}_{E}$, on a laminar wake is considered. The vorticity downstream of the body is $\omega$ and its development is described by the evolution equation (Batchelor 1967),

$$
\frac{\partial \boldsymbol{\omega}}{\partial t}+\left(\boldsymbol{U}_{E}+\boldsymbol{u}\right) \cdot \nabla \boldsymbol{\omega}=\boldsymbol{\omega} \cdot \nabla\left(\boldsymbol{U}_{E}+\boldsymbol{u}\right)+v \nabla^{2} \boldsymbol{\omega},
$$

where $\boldsymbol{u}$ is the flow perturbation due to the vorticity $\boldsymbol{\omega}=\boldsymbol{\nabla} \times \boldsymbol{u}$ in the wake and $v$ is the kinematic viscosity. When the external flow is uniform, $\boldsymbol{U}_{E}=\boldsymbol{U}$, the peak vorticity magnitude $\Omega$ in a laminar wake decreases by a combination of diffusion of vorticity towards the centreline, where it meets vorticity of the opposite sign, and viscous diffusion outwards into irrotational flow. In three-dimensional laminar flows, the local Reynolds number characterizing the wake, $R e_{w}=U_{w} Y / v$, decreases with downstream distance from the body essentially because the maximum flow perturbation $U_{w}$ decreases $\left(\sim x^{-1}\right)$ much faster than the rate at which the width of the wake, $Y\left(\sim x^{1 / 2}\right)$, increases. Thus, the flow is ultimately laminar. In planar flows, the Reynolds number characterizing the wake does not decrease with downstream distance. When $R e_{w} \geqslant 5$, the shed vortices ultimately generate the well-observed large alternately signed Boussinesq-Strouhal wake pattern. Many features of the mean flow in turbulent flows can be calculated by approximating the mean shear stress in terms of the mean velocity gradient and an eddy diffusivity $v_{e}$ that is constant across the wake but may vary along the wake. Keffer (1965) and Keffer et al. (1978) showed that this assumption is a good approximation in non-uniform external flows. As we shall demonstrate, however, in the presence of a positive external straining field, the local Reynolds number associated with the wake ultimately decreases so that in three-dimensional and planar cases the downstream flow is laminar.

Far enough downwind, the downstream flow is negligible compared to the external flow $\left(U_{w} \ll\left|\boldsymbol{U}_{E}\right|\right)$ and the vorticity equation (2.1) can be linearized. Vorticity is shed from the body, advected by the mean flow, and diffuses cross-stream, and, in the presence of an external strain parallel to vorticity components, the magnitude of the vorticity may be increased or decreased depending on the sign of the strain. The width of the wake $Y$ is typically much smaller than the characteristic streamwise distance over which the vorticity varies, so that streamwise gradients of vorticity are negligible compared to cross-stream gradients. Thus, in laminar flow, the evolution of the vorticity field is described by

$$
\frac{\partial \omega}{\partial t}+\boldsymbol{U}_{E} \cdot \nabla \omega=\omega \cdot \nabla \boldsymbol{U}_{E}+v \nabla_{H}^{2} \boldsymbol{\omega},
$$

where $\nabla_{H}^{2}=\partial^{2} / \partial y^{2}+\partial^{2} / \partial z^{2}$ is the two-dimensional Laplacian. The following analysis describes initial-value similarity solutions of (2.2), for a strained wake. 


\subsection{Planar converging and diverging flow}

\subsubsection{Velocity field in the wake}

The development of a steady laminar wake in the external straining field $\boldsymbol{U}_{E}=$ $(U(x),-\alpha y)$ is considered, where $\alpha$ is the strain rate and $U(x)=U_{0}+\alpha x$. Keffer (1965) and Elliott \& Townsend (1981) studied experimentally a slightly different problem, where the external strain field is generated by compression along the $y$-axis, expansion along the $z$-axis and a uniform flow along the $x$-axis; the development of solutions applicable to straining by this field are considered later in the paper. Under the former strain field, the vorticity equation (2.2) reduces to

$$
U_{0} \frac{\partial \omega}{\partial x}+\alpha x \frac{\partial \omega}{\partial x}-\alpha y \frac{\partial \omega}{\partial y}=v \frac{\partial^{2} \omega}{\partial y^{2}},
$$

where $\omega$ is the vorticity component perpendicular to the $(x, y)$-plane. For planar flow, (2.3) can be recast into the Boussinesq form (Boussinesq 1905)

$$
\frac{\partial \omega}{\partial \phi}=v \frac{\partial^{2} \omega}{\partial \psi^{2}}
$$

where the velocity potential and streamfunction corresponding to the external straining flow are $\phi=U_{0} x+\frac{1}{2} \alpha x^{2}-\frac{1}{2} \alpha y^{2}$ and $\psi=U_{0} y+\alpha x y$, respectively. The general solution, in terms of initial vorticity distribution $\omega_{0}(\psi)$ along a curve perpendicular to the streamlines located at $\phi=\phi_{0}$, is

$$
\omega(\psi, \phi)=\int_{-\infty}^{\infty} \frac{\omega_{0}\left(\psi^{\prime}\right)}{\sqrt{4 \pi v\left(\phi-\phi_{0}\right)}} \exp \left(-\frac{\left(\psi-\psi^{\prime}\right)^{2}}{4 v\left(\phi-\phi_{0}\right)}\right) \mathrm{d} \psi^{\prime} .
$$

Alternatively, a similarity method can also be used to solve (2.3). We demonstrate how they can equivalently be calculated from the exact solution (2.5). However, the Boussinesq transformation cannot be used for the axisymmetric case.

Recasting the vorticity equation in terms of the travel time $\tau$ from the origin simplifies (2.3) to

where

$$
\frac{\partial \omega}{\partial \tau}-\alpha y \frac{\partial \omega}{\partial y}=v \frac{\partial^{2} \omega}{\partial y^{2}}
$$

$$
\tau=\frac{1}{\alpha} \log \left(1+\frac{\alpha x}{U_{0}}\right) .
$$

The slowest decaying smoothest similarity solutions to (2.6) of the form $\omega=\Omega(\tau) \tilde{\omega}(\tilde{y})$ are sought, where $\Omega$ is the vorticity magnitude, $Y$ is the wake width and $\tilde{y}=y / Y(\tau)$. On substituting this form for $\omega,(2.6)$ becomes

$$
\left(\frac{Y^{2}}{v \Omega} \frac{\mathrm{d} \Omega}{\mathrm{d} \tau}\right) \tilde{\omega}-\left(\frac{Y}{v} \frac{\mathrm{d} Y}{\mathrm{~d} \tau}\right) \tilde{y} \frac{\mathrm{d} \tilde{\omega}}{\mathrm{d} \tilde{y}}-\left(\frac{Y^{2} \alpha}{v}\right) \tilde{y} \frac{\mathrm{d} \tilde{\omega}}{\mathrm{d} \tilde{y}}=\frac{\mathrm{d}^{2} \tilde{\omega}}{\mathrm{d} \tilde{y}^{2}} .
$$

In order that similarity solutions satisfy the above equation, we require

$$
\frac{Y^{2}}{v \Omega} \frac{\mathrm{d} \Omega}{\mathrm{d} \tau}, \quad \frac{Y}{v} \frac{\mathrm{d} Y}{\mathrm{~d} \tau}+\frac{Y^{2} \alpha}{v},
$$

to be constant. Thus, the width of the wake and the peak vorticity vary with $\tau$ according to

$$
Y^{2}=\frac{v}{\alpha}+\left(Y(0)^{2}-\frac{v}{\alpha}\right)\left(\frac{U_{0}}{U(x)}\right)^{2}
$$


and

$$
\frac{\Omega}{\Omega(0)}=\left(\frac{Y(0)}{Y}\right)^{\beta}\left(\frac{U_{0}}{U(x)}\right)^{\beta},
$$

where $Y(0)$ and $\Omega(0)$ are, respectively, the characteristic wake width and vorticity magnitude at the origin and $\beta$ is an unknown constant to be determined. Note that the first term in (2.9) is $-\beta$; if the second term in (2.9) is different from unity, the constant is absorbed into the wake width, but the final result is identical to that described below.

The similarity equation reduces to

$$
-\beta \tilde{\omega}-\tilde{y} \frac{\mathrm{d} \tilde{\omega}}{\mathrm{d} \tilde{y}}=\frac{\mathrm{d}^{2} \tilde{\omega}}{\mathrm{d} \tilde{y}^{2}} .
$$

Writing $\tilde{\omega}=f(\tilde{y}) \exp \left(-\frac{1}{2} \tilde{y}^{2}\right)$ transforms (2.12) into the Hermite equation,

$$
\frac{\mathrm{d}^{2} f}{\mathrm{~d} \tilde{y}^{2}}-\tilde{y} \frac{\mathrm{d} f}{\mathrm{~d} \tilde{y}}=-(\beta-1) f,
$$

whose general solution is

$$
f=A H_{\beta-1}(\tilde{y}),
$$

where $A$ is a constant and $H_{\beta-1}$ is the Hermite function. The choice of $\beta$ is determined by the initial vorticity field entering the flow. It is worth discussing the various solutions obtained, and how they relate to $\beta$.

When $\beta=0$, vorticity does not decay with distance, and

$$
\frac{\mathrm{d}^{2} \tilde{\omega}}{\mathrm{d} \tilde{y}^{2}}=-\tilde{y} \tilde{\omega}
$$

which gives

$$
\tilde{\omega}=\int_{0}^{\tilde{y}} \exp \left(-\frac{1}{2} \tilde{y}^{2}\right) \mathrm{d} \tilde{y} .
$$

The solution of $\beta=0$ corresponds to semi-infinitely deep layers of positive and negative vorticity being strained, whose integrated 'strength' does not change with distance. Further, when $\beta=1$, the vorticity field is

$$
\tilde{\omega}=\exp \left(-\frac{1}{2} \tilde{y}^{2}\right)
$$

and corresponds to the development of a source of vorticity. Clearly, for $\beta=0,1$ the assumptions of the linearization are not applicable because the flow perturbations are not small.

For the problem in hand, namely the large-scale straining of a line wake, the physical requirement is that the vorticity field is an odd function of $y$, and that $\tilde{\omega}$ does not change sign when $\tilde{y}>0$. The first constraint reduces to $\beta-1$ being an odd integer. The second constraint ensures that $\beta-1=1$ or $\beta=2$. As a consequence, the vorticity and velocity field are

$$
\tilde{\omega}=-\frac{1}{\left(\frac{1}{2} \pi\right)^{1 / 2}} \tilde{y} \exp \left(-\frac{1}{2} \tilde{y}^{2}\right), \quad \tilde{u}=-\frac{1}{\left(\frac{1}{2} \pi\right)^{1 / 2}} \exp \left(-\frac{1}{2} \tilde{y}^{2}\right),
$$

where $u_{x}=U_{w}(\tau) \tilde{u}(\tilde{y})$ and $U_{w}=\Omega(\tau)(\tau)$.

The volume flux associated with the wake is

$$
Q(x)=-\int_{-\infty}^{\infty} u \mathrm{~d} y=-2 \int_{0}^{\infty} y \omega \mathrm{d} y=-2 \Omega Y^{2} \int_{0}^{\infty} \tilde{y} \tilde{\omega} \mathrm{d} \tilde{y}=2 \Omega(x) Y(x)^{2},
$$




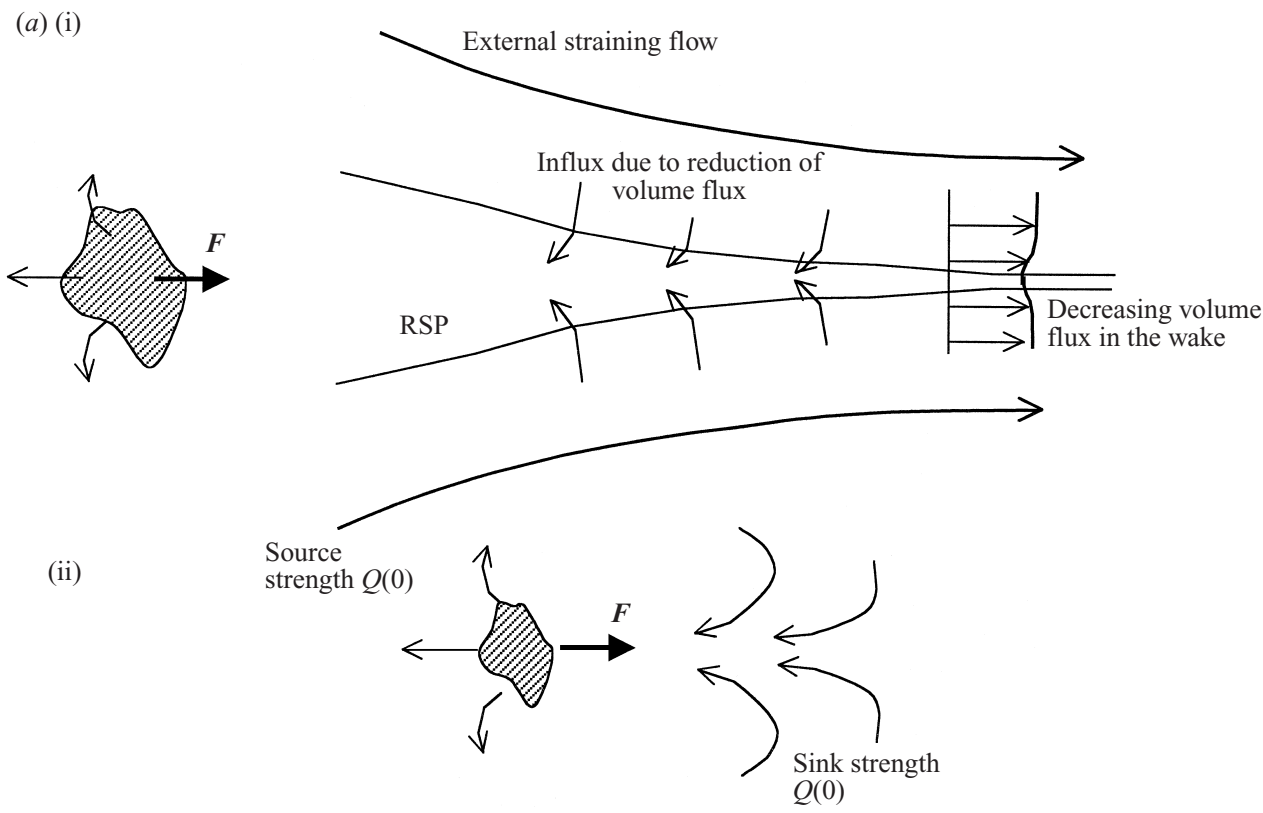

(b) (i)

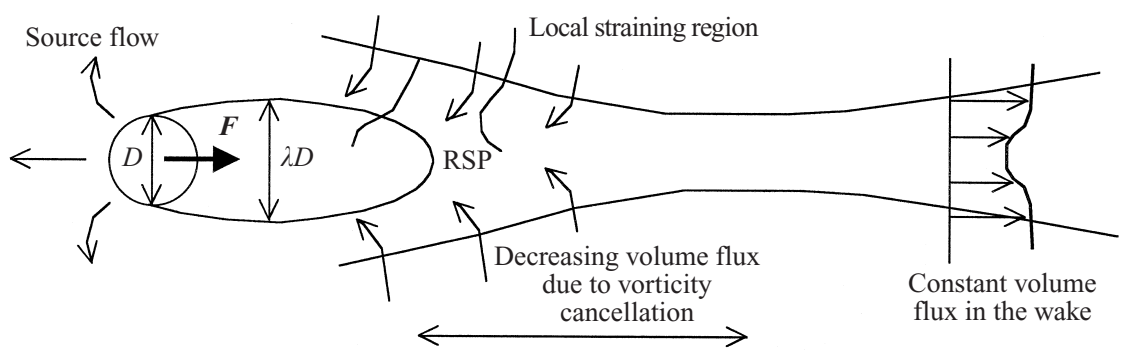

(ii)

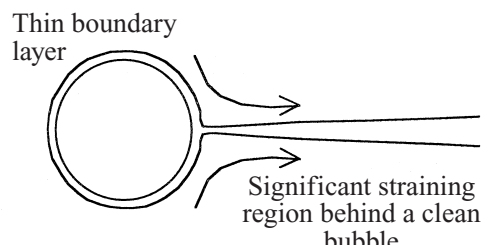

FIGURE 1. Schematic showing flow acceleration, vorticity cancellation and the wake associated with a rigid body in $(a)$ a weakly strained external flow, in (i) the near-field wake flow and (ii) the external perturbation velocity field; $(b)$ in a uniform ambient flow for (i) the region of the rear stagnation point with an attached wake and (ii) a bubble with an unseparated wake.

since the vorticity is an odd function of $y$. Thence, since $\beta=2$, from (2.10)

$$
\frac{Q(x)}{Q(0)}=\left(\frac{U_{0}}{U(x)}\right)^{2} \quad \text { where } \quad Q(0)=2 \Omega(0) Y^{2}(0) .
$$

An important point is that the change in the volume flux in the wake is independent of viscosity, and depends only on the strain rate and distance $x$. Figure 1(a) shows a 
schematic of the essential features of the near- and far-field flow in a weakly strained external flow.

We observe two different types of wake development, depending on whether $\alpha$ is positive or negative. When the flow converges $(\alpha>0)$, the width of an initially narrow wake $\left(\tilde{Y}(0)=Y(0) /(v /|\alpha|)^{1 / 2} \ll 1\right)$ rapidly increases with downstream distance owing to enhanced diffusion and ultimately tends to a constant value $(v / \alpha)^{1 / 2}$ and the peak vorticity $\Omega$ decreases much more rapidly with distance from the body than in uniform flow,

$$
Y \rightarrow(v / \alpha)^{1 / 2}, \quad \frac{\Omega(x)}{\Omega(0)} \rightarrow \frac{\tilde{Y}^{2}(0)}{\left(U(x) / U_{0}\right)^{2}} .
$$

When the wake is initially wide, $\tilde{Y}(0) \gg 1$, the wake width decreases owing to the convergence of the flow and diffusional effects are initially small, so that

$$
Y \sim \frac{\tilde{Y}(0)}{U(x) / U_{0}}, \quad \frac{\Omega(x)}{\Omega(0)} \sim 1,
$$

but the wake width ultimately tends to a constant width which is described by (2.21). Note that the peak velocity decays as $U_{w} \sim U_{w 0} /\left(\alpha x / U_{0}\right)^{2}$ with downstream distance in strained flows, much more rapidly than in unstrained flows where $U_{w} \sim$ $U_{w 0} /\left(2 v x / U_{0} Y(0)^{2}\right)^{1 / 2}$

When $\alpha<0$, the straining velocity field tends to zero at the stagnation surface $x=-U_{0} / \alpha$, and the wake width increases downstream without limit. The asymptotic forms of $Y(x)$ and $\Omega(x)$ along streamlines vary considerably depending on whether the initial width $Y(0)$ is large or small compared to the viscous-straining asymptotic scale $(v /|\alpha|)^{1 / 2}$. When $\tilde{Y}(0) \ll 1$, the vorticity tends to a constant value, so that

$$
Y \rightarrow \frac{(v /|\alpha|)^{1 / 2}}{U(x) / U_{0}}, \quad \frac{\Omega(x)}{\Omega(0)} \rightarrow \tilde{Y}(0)^{2}(\ll 1),
$$

while for $\tilde{Y}(0) \gg 1$, the wake development is independent of $v$; its width grows rapidly with distance and the vorticity is comparable to its initial value,

$$
Y \rightarrow \frac{Y(0)}{U(x) / U_{0}}, \quad \frac{\Omega(x)}{\Omega(0)} \rightarrow 1 .
$$

Therefore, the peak velocity $\left(U_{w} / U_{w 0} \sim U_{0} / U(x)\right)$ and volume flux $(Q / Q(0) \sim$ $\left.\left(U_{0} / U(x)\right)^{2}\right)$ increase with distance. The linearization of the vorticity equation is therefore only valid within a distance $O\left(U_{0} /|\alpha|\right)$ from $x=0$, beyond which the nonlinear convection induced by the wake must be considered.

These similarity solutions are consistent with the exact solution to the linearized equation (2.5), as demonstrated in Appendix A.

\subsubsection{Wake effects on the external velocity field}

When $\alpha>0$, the similarity solution is valid far downstream of the body and we proceed to study the external velocity field in more detail. The flow induced by the wake is equivalent to a distribution of line sources of strength $m(x)$ where

$$
m(x)=\frac{\mathrm{d} Q}{\mathrm{~d} x}=-\frac{2 Q(0) \alpha / U_{0}}{\left(1+\alpha x / U_{0}\right)^{3}} .
$$

Since $\alpha>0$, the strength of the sources tends to zero and their cumulative effect on the external flow is finite. The external potential flow generated by the wake is 
determined by integrating the cumulative effect of the distribution of sources, i.e.

$$
\boldsymbol{u}(x, y)=\int_{0}^{\infty} \frac{\mathrm{d} Q}{\mathrm{~d} x^{\prime}} \boldsymbol{S}\left(x^{\prime}, x, y\right) \mathrm{d} x^{\prime},
$$

where

$$
\boldsymbol{S}\left(x^{\prime}, x, y\right)=\frac{1}{2 \pi} \frac{\left(x-x^{\prime}, y\right)}{\left(x-x^{\prime}\right)^{2}+y^{2}}
$$

is the flow induced at $(x, y)$ by a unit source located at $\left(x^{\prime}, 0\right)$. Integrating by parts, (2.26) shows that the flow consists of a far-field sink of strength $Q(0)$ and a distribution of dipoles of strength $Q\left(x^{\prime}\right)$ located along the $x$-axis:

$$
\boldsymbol{u}(x, y)=-Q(0) \boldsymbol{S}(0, x, y)-\int_{0}^{\infty} Q\left(x^{\prime}\right) \frac{\mathrm{d} \boldsymbol{S}\left(x^{\prime}, x, y\right)}{\mathrm{d} x^{\prime}} \mathrm{d} x^{\prime} .
$$

Thus, the external flow induced by the source generated by the body and the wake is due to a dipole of strength

$$
2 \pi \mu_{x}=-\int_{0}^{\infty} Q\left(x^{\prime}\right) \mathrm{d} x^{\prime}=-\frac{Q(0) U_{0}}{\alpha},
$$

because the sink at the origin cancels with the source generated by the body.

By integrating (2.26) and (2.27), the external velocity field $\boldsymbol{u}=\left(u_{x}, u_{y}\right)$ is found to be

$$
\begin{aligned}
u_{x}=\frac{Q(0) U_{0}^{2}}{\pi \alpha^{2}}[ & -\frac{\left(3 y^{2}+\left(U_{0} / \alpha+x\right)^{2}\right)\left(x+U_{0} / \alpha\right)}{\left(\left(U_{0} / \alpha+x\right)^{2}+y^{2}\right)^{3}} \log \left(\frac{\alpha}{U_{0}}\left(x^{2}+y^{2}\right)^{1 / 2}\right) \\
& -\frac{\alpha / U_{0}\left(\left(U_{0} / \alpha+x\right)^{2}-y^{2}\right)}{\left(\left(U_{0} / \alpha+x\right)^{2}+y^{2}\right)^{2}}-\frac{\alpha^{2} / U_{0}^{2}\left(x+U_{0} / \alpha\right)}{2\left(\left(U_{0} / \alpha+x\right)^{2}+y^{2}\right)} \\
& \left.+\frac{-y^{4}+3 y^{2}\left(x+U_{0} / \alpha\right)^{2}}{\left(\left(U_{0} / \alpha+x\right)^{2}+y^{2}\right)^{3}}\left(\frac{1}{2} \pi-\tan ^{-1} \frac{-x}{y}\right)\right]
\end{aligned}
$$

and

$$
\begin{aligned}
u_{y}=- & \frac{Q(0) y U_{0}^{2}}{\pi \alpha^{2}}\left[-\frac{3\left(U_{0} / \alpha+x\right)^{2}-y^{2}}{\left(\left(U_{0} / \alpha+x\right)^{2}+y^{2}\right)^{3}} \log \left(\frac{\alpha}{U_{0}}\left(x^{2}+y^{2}\right)^{1 / 2}\right)+\frac{2(U / \alpha+x) \alpha / U_{0}}{\left(\left(U_{0} / \alpha+x\right)^{2}+y^{2}\right)^{2}}\right. \\
& \left.+\frac{\alpha^{2} / U_{0}^{2}}{2\left(\left(U_{0} / \alpha+x\right)^{2}+y^{2}\right)}+\frac{3\left(U_{0} / \alpha+x\right)^{2}-y^{2}}{\left(\left(U_{0} / \alpha+x\right)^{2}+y^{2}\right)^{3}}\left(\frac{1}{2} \pi-\tan ^{-1} \frac{-x}{y}\right)\right] .
\end{aligned}
$$

Thus, the external flow induced by the wake is equivalent to a sink of strength $Q(0)$ (identified as the third term on the right-hand side of the above expression) and a dipole of strength $-Q(0) U_{0} / \pi \alpha$ located at $x=-U_{0} / \alpha$ (identified as the second term on the right-hand side of the above expression), as well as the usual source field located at the origin. The combination of an apparent sink located in front of the body, and a source at the origin yields a far-field dipolar flow (see figure $1 a$, ii). Thus, the external flow induced by the wake and body is equivalent to a dipole of strength $\mu_{x}=-Q(0) U_{0} / 2 \pi \alpha$, a result identical to (2.29) which was obtained without direct calculation of the far-field flow.

\subsection{Axisymmetric flow}

The effect of a laminar wake is examined in an axisymmetric straining field $\boldsymbol{U}_{E}=$ $(U(x),-\alpha r)$, defined in cylindrical polar coordinates $(x, r, \theta)$, where $U(x)=U_{0}+2 \alpha x$. The dynamical difference between planar and axisymmetric flows is that the straining 
The disappearance of laminar and turbulent wakes in complex flows

field compresses the vortical elements which would tend to increase the strength of the vorticity close to the centreline. The development of an axisymmetric flow, due to an azimuthal vorticity field, is considered. The vorticity equation describing the development of $\omega_{\theta}$, the azimuthal component of vorticity, is

$$
\boldsymbol{U}_{E} \cdot \nabla \omega_{\theta}=\frac{\boldsymbol{U}_{E} \cdot \hat{\mathbf{r}} \omega_{\theta}}{r}+v\left(\frac{1}{r} \frac{\partial}{\partial r}\left(r \frac{\partial \omega_{\theta}}{\partial r}\right)-\frac{\omega_{\theta}}{r^{2}}\right) .
$$

The development of the flow is expressed in terms of $\Gamma=\omega_{\theta} / r$ which is proportional to the circulation around a vortical element and is conserved during axisymmetric stretching by the straining flow. The downstream development of $\Gamma$ is described by

$$
\frac{\partial \Gamma}{\partial \tau}-\alpha r \frac{\partial \Gamma}{\partial r}=v\left(\frac{\partial^{2} \Gamma}{\partial r^{2}}+\frac{3}{r} \frac{\partial \Gamma}{\partial r}\right)
$$

where the travel time from the origin is

$$
\tau=\frac{1}{2 \alpha} \log \left(1+\frac{2 \alpha x}{U_{0}}\right) .
$$

Similarity solutions of the form $\Gamma=G(\tau) \tilde{\Gamma}(\tilde{r})$, where $\tilde{r}=r / R(\tau)$, are sought. The above equation is transformed to

$$
\left(\frac{R^{2}}{v G} \frac{\mathrm{d} G}{\mathrm{~d} \tau}\right) \tilde{\Gamma}-\left(\frac{R}{v} \frac{\mathrm{d} R}{\mathrm{~d} \tau}\right) \tilde{r} \frac{\mathrm{d} \tilde{\Gamma}}{\mathrm{d} \tilde{r}}-\left(\frac{R^{2} \alpha}{v}\right) \tilde{r} \frac{\mathrm{d} \tilde{\Gamma}}{\mathrm{d} \tilde{r}}=\left(\frac{\mathrm{d}^{2} \tilde{\Gamma}}{\mathrm{d} \tilde{r}^{2}}+\frac{3}{\tilde{r}} \frac{\mathrm{d} \tilde{\Gamma}}{\mathrm{d} \tilde{r}}\right) .
$$

The dimensionless coefficients

$$
\frac{R^{2}}{v G} \frac{\mathrm{d} G}{\mathrm{~d} \tau}, \quad \frac{R}{v} \frac{\mathrm{d} R}{\mathrm{~d} \tau}+\frac{R^{2} \alpha}{v},
$$

are constant, from which the width of the wake and strength of circulation are found to vary (in a similar fashion to the planar problem) as

$$
R^{2}=\frac{v}{\alpha}+\left(R(0)^{2}-\frac{v}{\alpha}\right) \exp (-2 \alpha \tau)
$$

and

$$
\frac{G}{G(0)}=\left(\frac{R(0)}{R}\right)^{\beta} \exp (-\alpha \beta \tau)
$$

The similarity solution satisfies

$$
\tilde{r} \frac{\mathrm{d}^{2} \tilde{\Gamma}}{\mathrm{d} \tilde{r}^{2}}+\left(3+\tilde{r}^{2}\right) \frac{\mathrm{d} \tilde{\Gamma}}{\mathrm{d} \tilde{r}}+\beta \tilde{\Gamma} \tilde{r}=0 .
$$

Writing $w=-\frac{1}{2} \tilde{r}^{2}$, transforms the above equation to

$$
w \frac{\mathrm{d}^{2} \tilde{\Gamma}}{\mathrm{d} w^{2}}+(2-w) \frac{\mathrm{d} \tilde{\Gamma}}{\mathrm{d} w}-\frac{1}{2} \beta \tilde{\Gamma}=0 .
$$

The above is known as Kummer's differential equation and the general solution is expressed as

$$
\tilde{\Gamma}=M\left(2, \frac{1}{2} \beta,-\frac{1}{2} \tilde{r}^{2}\right)=\exp \left(-\frac{1}{2} \tilde{r}^{2}\right) M\left(\frac{1}{2}(4-\beta), \frac{1}{2} \beta, \frac{1}{2} \tilde{r}^{2}\right),
$$

where $M$ is the confluent hypergeometric function (see Arfken 1985 p. 753). In the limit of $\tilde{r} \rightarrow 0$, the circulation tends to $\Gamma \sim \tilde{r}^{n}$. Substituting $\Gamma \sim \tilde{r}^{n}$ into (2.37), shows 
that $4+n=0$ and $\beta+n=0$. Thus, $\beta=4$ and the vorticity field is determined from

$$
\omega(\tau, r)=\frac{G(\tau)}{\tilde{r}} \exp \left(-\frac{1}{2} \tilde{r}^{2}\right) M\left(0,2, \frac{1}{2} \tilde{r}^{2}\right)
$$

The volume flux in the wake is related to the weighted integral of the vorticity as follows:

$$
Q(x)=-\int_{0}^{\infty} u_{x} \mathrm{~d}\left(\pi r^{2}\right)=-\int_{0}^{\infty} \pi r^{2} \omega \mathrm{d} r=\pi R^{4} G(\tau) \int_{0}^{\infty} \tilde{r}^{3} \tilde{\Gamma} \mathrm{d} \tilde{r} .
$$

Thence, from (2.39), the flow volume flux decays as

$$
\frac{Q(x)}{Q(0)}=\exp (-\beta \alpha \tau)=\frac{1}{\left(1+2 \alpha x / U_{0}\right)^{2}}
$$

with distance downwind of the body. The external flow induced by the wake is equivalent to a distribution of point sources of strength $m(x)$ where

$$
m(x)=\frac{\mathrm{d} Q}{\mathrm{~d} x}=-\frac{4 Q(0) \alpha / U_{0}}{\left(1+2 \alpha x / U_{0}\right)^{3}} .
$$

The external potential flow induced by the wake is determined by the cumulative effect of sources located along the centreline and is described by (2.26), with $\boldsymbol{S}$ now representing the flow due to a point source located at the point $\left(x^{\prime}, 0\right)$ (expressed in cylindrical coordinates): $\boldsymbol{S}\left(x^{\prime}, x, r\right)=\left(x-x^{\prime}, r\right) / 4 \pi\left(\left(x-x^{\prime}\right)^{2}+r^{2}\right)^{3 / 2}$. Here, the far-field flow is again dipolar and has strength

$$
4 \pi \mu_{x}=-\int_{0}^{\infty} Q\left(x^{\prime}\right) \mathrm{d} x^{\prime}=-\frac{Q(0) U_{0}}{2 \alpha} .
$$

The far-field flow could potentially be obtained by integrating (2.26), but the lengthy expressions which result have no bearing on the physics of the problem and are not included here.

\subsection{Strained turbulent wakes}

The prediction of the decay in the vorticity and the volume flux of the wake behind a rigid body is in accordance with the experimental measurements of Keffer (1965) who studied the spreading of a turbulent planar wake characterized by $R e \simeq 1700$ in a converging-straining flow, and measured the turbulent and mean structure of the downstream wake. An interesting feature of the latter experiments, confirmed by our theory, was that $U_{w}$ was effectively constant. Although the external flow has a similar effect to that considered in this paper, it is three-dimensional, i.e.

$$
\boldsymbol{U}_{E}=\left(U_{0},-\alpha x, \alpha z\right),
$$

so that the streamwise component of the mean velocity is unchanged with distance because there is a diverging strain in the $z$-direction. The similarity solution proposed by Keffer (1965) (which effectively assumed a constant eddy viscosity) differs from that developed in this paper. His method led to the prediction that the width and mean speed in the wake tended to zero exponentially, in conflict with experimental observations.

Using the more systematic method applied here, similarity solutions can be constructed with the flow field (2.44) assuming the eddy viscosity remains approximately constant even when the mean vorticity decays. Physically, this can be explained partially by the amplification of the turbulent velocity caused by the mean flow 


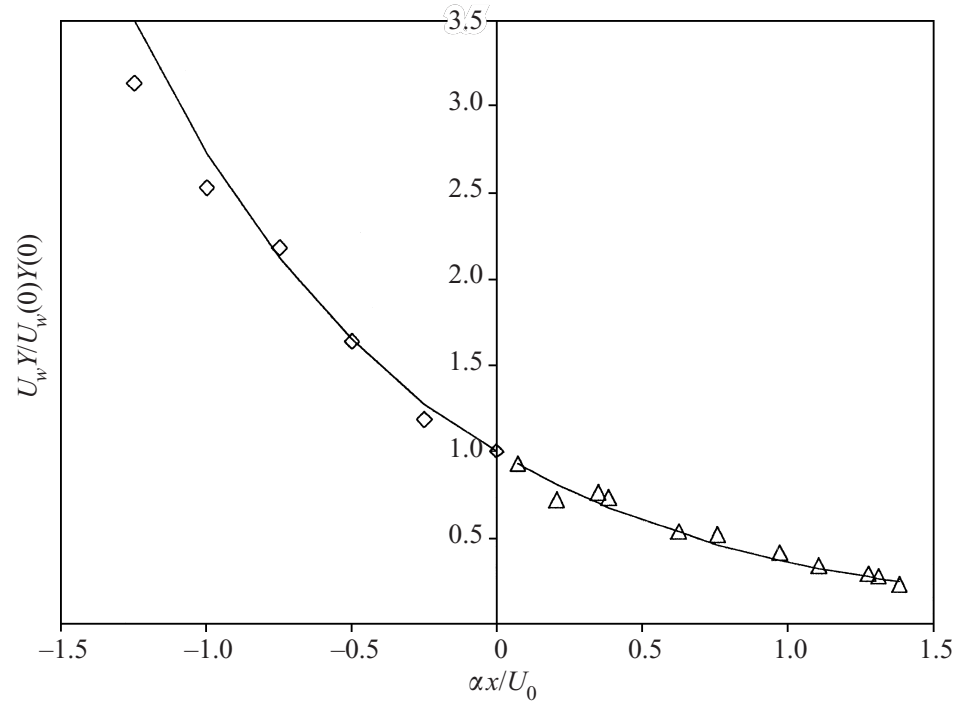

FIGURE 2. Comparison between the experimental measurements of the volume flux in the planar turbulent wake of a cylinder, in the three-dimensional straining flow (2.44), and a similarity solution based on a constant eddy viscosity assumption. The triangles correspond to the measurements of the wake width $Y$ and characteristic deficit velocity $U_{w}$, taken from figure 3 of Keffer (1965) for $\alpha>0$, while the diamonds correspond to the measurements (taken from table 1) of Elliott \& Townsend (1981) for $\alpha<0$. The strain rate is determined in Keffer's experiments from the geometry of the duct, whereas Elliott \& Townsend (1981) made measurements of the strain rate (which varied with streamwise distance) and a value of $-2.5 \mathrm{~m}^{-1}$ is assumed for $\alpha / U_{0}$. In the present notation, $x=0$ corresponds to the start of the distorting duct. The solid curve corresponds to the theoretical prediction $U_{w} Y / U_{w}(0) Y(0)=\exp \left(-\alpha x / U_{0}\right)$.

straining of the turbulent vorticity. The width of a turbulent shear layer in the $y$-direction or a scalar plume are not very sensitive to the variation of the eddy viscosity or eddy diffusivity (cf. Keffer et al. 1978). It is found that the width of the wake increases according to $(2.10)$ and the mean vorticity decreases according to $\Omega(x)=\Omega(0) \exp \left(-\alpha x / U_{0}\right)(Y(0) / Y(x))^{2}$. According to this calculation, the width of the wake tends to a constant value and the product of velocity defect $U_{w}$ and width $Y$ varies as $U_{w} Y / U_{w}(0) Y(0)=\exp \left(-\alpha x / U_{0}\right)$, independent of the magnitude of the eddy viscosity. This provides a means of testing the analysis without fitting or prescribing the eddy diffusivity. Figure 2 shows a comparison between the experimental measurements of $Q=U_{w} Y$ by Keffer (1965) (for $\alpha>0$ ) and Elliott \& Townsend (1981) (for $\alpha<0)$ and the predictions based on our similarity solutions. The agreement is good.

\section{Wake vorticity, the external flow and force on bodies}

The force and moment on a body, without and with an external strain field, can always be related to the momentum flux of the external flow which is defined by the ambient uniform and straining fields and the source and dipole perturbation to the external field (see figure 1) (Magnaudet \& Eames 2000). In a uniform flow of speed $U_{0}$, the force on the body, $F$, is equal to the momentum flux in the wake $\left(2 \rho Q(0) U_{0}\right)$ less the propulsive force produced by the potential flow source field around the body $\left(-\rho Q(0) U_{0}\right)$ (see Batchelor 1967). For instance, the total force acting on a planar 
obstacle in a uniform flow, such as a cylinder, is

$$
F_{0}=\frac{1}{2} \rho C_{D} D U_{0}^{2}=\rho Q(0) U_{0}
$$

where $C_{D}$ is the drag coefficient and $D$ is the projected width of the planar body (or diameter in the case of a cylinder). Note that this requires the Reynolds number associated with the flow past the body and the wake to be large, i.e.

$$
R e=U_{0} D / v \gg 1, \quad R e_{W}=\left(Q(0) / U_{0} D\right) R e \gg 1 .
$$

The latter constraint (which is usually omitted) ensures that the wake is turbulent and it is important when considering the force on bubbles or fluid volumes which are shedding vorticity (see below). In a uniform flow, the interactions in the external flow between the uniform flow, and the dipole (and higher-order) fields do not affect the drag. However, in a straining field these need to be considered. When the external straining field is negligible, a bluff body, for instance a circular cylinder, induces an external dipole field, that for an inviscid flow is

$$
M_{0}^{(I)}=-\frac{1}{4} U_{0} \pi D^{2} .
$$

However, in high-Reynolds-number viscous flows, the dipole strength is determined by the flow around the separated streamlines. It is larger than in inviscid flows because the flow separation occurs at an angle $\theta_{s}$ from the upstream direction (Parkinson \& Jandali 1970), so that

$$
M_{0}^{(S)} \approx-\frac{1}{4} U_{0} \pi D^{2}-Q(0) D \cos \beta,
$$

where $\beta$ is associated with the separation angle $\theta_{s}$ and is approximately $90^{\circ} \pm 10^{\circ}$. The dipole strength was calculated from the potential flow model of Parkinson \& Jandali (1970) in which sources outside the cylinder produce the same effect as a separated wake. These determine the magnitude of the angle $\beta$. Since $Q(0)$ is related to the drag coefficient,

$$
M_{0}^{(S)} \approx-\frac{1}{4} U_{0} \pi D^{2}\left[1+\frac{2 C_{D}}{\pi} \cos \beta\right] .
$$

Typical values are $\beta=80^{\circ}, C_{D}=1.09$, so that $1+\left(2 C_{D} / \pi\right) \cos \beta \approx 1.12$, so that $M_{0}^{(S)} / U_{0} D^{2}$ is in fact approximately equal to $M_{0}^{(I)} / U_{0} D^{2}$. The source component of this dipole is mainly produced by the diverging flow near the front stagnation and the sink component by the converging flow at the rear stagnation point (RSP) of the wake (see figure 1). Note that there is a marked transition over a distance $L_{w}$ (of order of the body size $D$ ) as this flow region is dominated by the straining motion close to the stagnation streamline, which reduces the volume flux in the wake by the mechanism described in $\S 2$.

For the case of flow past a slender body, the wake width is much larger than the straining-viscous lengthscale near the RSP (i.e. $\left.Y(0) \gg(v / \alpha)^{1 / 2}\right)$ so that total vorticity cancellation is small. However, it is non-zero and can be calculated along the attached streamline as the vorticity generated along the body surface adjusts to the shear-free condition along the attached streamline. The volume flux in the laminar wake of a flat plate, $Q_{w}(x)$, downstream of the RSP (see Appendix B) is

$$
Q_{w}(x)=Q(0)+\frac{1}{U_{0}} \int_{-\infty}^{\infty}\left(u-U_{0}\right)^{2} \mathrm{~d} y,
$$

which decreases with downstream distance and tends to the far-field value of $Q(0)$. The detailed calculations of the mean turbulent flow past a flat plate by Neish \& Smith 
(1988) show that the displacement thickness (which is proportional to the volume flux) decreased by $10 \%$ from the value at the RSP. Associated with the decrease in the volume flux is a distribution of sinks which tend to increase the far-field dipole strength associated with flow past the plate, which is dominated by the volume flux increasing along the length of the plate and is estimated to be $O\left(U_{0} L^{2} R e^{-1 / 2}\right)$. A relatively larger decrease in the wake volume flux also occurs downwind of the RSP of bluff bodies, although this transition region is not included in the near-field analysis of Parkinson \& Jandali (1970) or other investigations.

This vorticity cancellation is much stronger in flow around a bluff body with thin wakes, such as high-Reynolds-number flow around a clean spherical bubble. The flow around a bubble of radius $a$ was shown by Moore (1963) to be characterized by a thin boundary layer of thickness $O\left(a R e^{-1 / 2}\right)$ (where $\left.R e=2 a U_{0} / v\right)$, which does not separate from the bubble surface, and the boundary-layer flow is a weak perturbation to the primary irrotational flow. The analysis developed in this paper may be applied to examine the significant changes in the volume flux in the wake of a bubble. Downstream of the bubble and close to the attached streamline, to leading order, the wake is subject to the potential straining flow $U_{0}\left(1-(a / x)^{3},-\frac{3}{2} y(a / x)^{4} / a\right)$, which is expressed in cylindrical coordinates, where $x$ is the downstream distance, $y$ is the cross-stream distance. Similarity solutions may be developed which show that the volume flux in the wake, $Q_{w}$, at distances $x_{0}$ and $x$ from the bubble centre are related through

$$
\frac{Q_{w}\left(x_{0}\right)}{Q_{w}(x)}=\left(\frac{1-a^{3} / x^{3}}{1-a^{3} / x_{0}^{2}}\right)^{2} .
$$

Moore (1963) calculated the flow field in the near wake region but not the wake flow far downstream of the bubble. The volume flux at the start of the wake region, $x_{B}=$ $a+O\left(a R e^{-1 / 6}\right)$, is calculated from Moore's analysis to be $Q_{B}=Q_{w}\left(x_{B}\right)=24 U_{0} \pi a^{2} R e^{-1}$. According to (3.7), the volume flux in the wake at a distance $x$ decreases rapidly with distance, ultimately tending to

$$
Q_{w}(x \rightarrow \infty)=Q(0)=24 U_{0} \pi a^{2} R e^{-1} O\left(R e^{-1 / 3}\right),
$$

far downwind of the bubble. Thus, the wake volume flux associated with any clean bubble is significantly less than the wake flux at the RSP because of the significant straining motions induced by the leading-order potential straining flow generated in this region. Since the ratio of the wake width $Y(0)$ to the viscous-straining lengthscale $(v / \alpha)^{1 / 2}\left(\sim O\left(a R e^{-1 / 2}\right)\right)$, is $Y(0) /(v / \alpha)^{1 / 2} \sim O(1)$, the reduction of the wake vorticity and the volume flux is much greater than for a bluff body where $Y(0) /(v / \alpha)^{1 / 2} \sim R e^{1 / 2}$. In fact, as we have shown, the far-wake momentum deficit for a bubble is much less (by a factor $R e^{-1 / 3}$ ) than the drag force, i.e. $F_{0} \gg \rho Q(0) U_{0}$. This is a fundamental limitation of Betz's (1925) result that has not previously been pointed out. For bubbles, the flow approximates to potential flow, except in the boundary layer, and the drag is related to the weak viscous stresses in the potential flow (Batchelor 1967 p. 368). The same analysis can be extended to eddies or vortex rings with narrow wakes (Turner 1973).

In the presence of weak strain, where $0<\alpha D / U_{0} \ll 1$, the analysis of $\S 2$ shows that the wake structure completely changes by $O(1)$ over a long distance $O\left(U_{0} / \alpha\right)$ from the body. Thus, the addition of weak strain is a singular perturbation to the flow far from the body. However, near the body and in the near wake, the flow is not affected (at least to $O\left(\alpha D / U_{0}\right)$ ). Although the wake downstream disappears, the analysis of $\S 2.2$ shows that the momentum flux in the wake near the body is equal to 
the contribution to the change of the momentum flux in the external flow. Since the pressure distribution over the body is changed by $O\left(\alpha D / U_{0}\right)$, the force on the body is changed slightly by this amount. In addition to changing the distribution of the momentum flux deficit, the weak strain field also changes the force on the body which is determined by the combined action of the strain field $(\mathrm{d} U / \mathrm{d} x)$ and the dipole field $M . M$ is a combination of the dipole in the absence of the strain field, $M_{0}^{(S)}$ (e.g. given for separated flow by (3.4)), and $2^{n} \pi \mu_{x}$, the dipole associated with the disappearing wake (see (2.29) and (2.43)) (where $n=1,2$ for planar and axisymmetric flows):

$$
M \approx M_{0}^{(S)}+2^{n} \pi \mu_{x}=M_{0}^{(S)}-Q(0) U_{0} / 2^{(n-1)} \alpha .
$$

Since the source and dipole field in the external flow are weak perturbations to the imposed external flow (and the external flow is irrotational) and since, with finite external strain, the far-field wake disappears algebraically with downwind distance, the total force is given exactly by

$$
F=-\rho M \frac{\mathrm{d} U}{\mathrm{~d} x} .
$$

Equations (3.9) and (3.10) show that the total force, for quite moderate strain rates $\left(0<\alpha D / U_{0}<1\right)$, is equal to the viscous force in uniform flows plus the inviscid force due to the straining field, i.e.

$$
F \approx-\rho M_{0}^{(I)} \frac{\mathrm{d} U}{\mathrm{~d} x}+\rho Q(0) U_{0},
$$

since, as explained below (3.4), $M_{0}^{(S)} \approx M_{0}^{(I)}$.

This is consistent with the results of the computations reviewed by Magnaudet \& Eames (2000) which showed that the 'added-mass' force and the drag force for uniform flow can be superimposed. This approximation is now a standard assumption in two-phase flow computations (e.g. Kowe et al. 1988) and is found to agree with data even when the added-mass term is comparable to the drag term and when a high $(\sim 20 \%)$ proportion of the fluid is occupied by moving objects (which is consistent with the wakes effectively disappearing) (e.g. Couet, Brown \& Hunt 1991). Of course, when $\alpha D / U_{0}=O(1)$, the strain affects the near-field flow and (3.11) is no longer valid (Graham 1980).

\section{Disappearing wakes in complex flows}

\subsection{Effect of piecewise uniform strain on a laminar wake}

The strain rate acting on the wake of a body, as it is advected past a series of bodies, typically varies with distance along the streamline. In order to gain insight into the effect of non-uniform strain, we proceed to apply the similarity analysis to examine the fundamental changes in a viscous laminar wake due to an alternately signed straining field. Of course, wakes are often split into two when they meet a downwind body in a similar way as a scalar 'plume' is split (Hunt \& Mulhearn 1973).

The development of a laminar wake, of initial vorticity $\Omega_{0}$ and width $Y_{0}($ at $x=0)$, is perturbed by alternate positive and negative uniform strain. The strain rate is uniform within streamwise segments of the flow, within the regions $\left[0, l_{1}\right],\left[l_{1}, l_{1}+l_{2}\right]$, $\left[l_{1},+l_{2}, 2 l_{1}+l_{2}\right], \ldots$. The wake spreading in this idealized problem is defined by $U_{0}$, the velocity at the origin, $U_{1}$, the velocity at the end of the first and odd numbered regions, and $U_{2}$, the velocity at the end of the second and even numbered straining regions. When the strain is alternately positive and negative, and the mean speed at 
$x=l_{1}+l_{2}, 2\left(l_{1}+l_{2}\right), \ldots$ adjusts to the value at $x=0, U_{2} / U_{0}=1$ and $U_{1} / U_{0}=\mathscr{S}$. The strain rates in the odd and even numbered regions are thus $\alpha_{1}=(\mathscr{S}-1) U_{0} / l_{1}$ and $\alpha_{2}=-(\mathscr{S}-1) U_{0} / l_{2}$, respectively. The development of the width and vorticity magnitude corresponding to the similarity solutions may be recast in terms of $U_{i}$ using (2.10) and (2.11).

In the first straining region, the results of $\S 2.1$ show that the dimensionless wake width and vorticity magnitude are

$$
\tilde{Y}_{1}^{2}=1+\frac{1}{\mathscr{S}^{2}}\left(\tilde{Y}_{0}^{2}-1\right), \quad \frac{\Omega_{1}}{\Omega_{0}}=\frac{\tilde{Y}_{0}^{2}}{\tilde{Y}_{1}^{2}} \frac{1}{\mathscr{S}^{2}},
$$

where $\tilde{Y}=Y /\left(v /\left|\alpha_{1}\right|\right)^{1 / 2}$. There are two limiting cases corresponding to a narrow initial wake: $\tilde{Y}_{0} \ll 1$, where diffusion controls the increase in the wake width so that $\tilde{Y}_{1} \rightarrow 1\left(\gg \tilde{Y}_{0}\right)$, and $1 \ll \tilde{Y}_{0} \ll \mathscr{S}$, where the wake width decreases to $\tilde{Y}_{1} \rightarrow 1\left(\ll \tilde{Y}_{0}\right)$ owing to diffusive effects being negligible and the converging flow causes the wake width to decrease. For a narrow initial wake, the vorticity magnitude decreases owing to vorticity cancellation and $\Omega_{1} / \Omega_{0}=\tilde{Y}_{0}^{2} / \mathscr{S}^{2}$. By contrast, for an initially wide wake, $\tilde{Y}_{0} \gg \mathscr{S}$, the wake width decreases owing to the positively straining flow and $\tilde{Y}_{1} \rightarrow \tilde{Y}_{0} / \mathscr{S}$ and the change in the vorticity magnitude is negligible because diffusion is weak, i.e. $\Omega_{1} / \Omega_{0} \rightarrow 1$.

In the second negatively straining region, the wake width can be expressed exactly in terms of its initial width,

$$
\tilde{Y}_{2}^{2}-\tilde{Y}_{0}^{2}=\left(\mathscr{S}^{2}-1\right)\left(1+l_{2} / l_{1}\right) .
$$

When the width of the initial wake is small $\left(\tilde{Y}_{0} \ll 1\right)$, the final wake width is dominated by diffusive effects and $\tilde{Y}_{2} \sim \mathscr{S}\left(1+l_{2} / l_{1}\right)^{1 / 2}$, independent of the initial wake width. An initially wide wake returns back to its value, $\tilde{Y}_{2}=\tilde{Y}_{0}$. The vorticity in this region is

$$
\frac{\Omega_{2}}{\Omega_{0}}=\frac{\tilde{Y}_{0}^{2}}{\tilde{Y}_{2}^{2}} .
$$

This indicates that while the volume flux in the wake changes rapidly in the positively and negative strained regions, the volume flux is finally preserved after the application of the two alternate uniform straining regions, providing that the mean flow has recovered to its initial value (i.e. $U_{2} / U_{0}=1$ ). Thus, when the width of the wake is initially small, the wake widens dramatically to a non-dimensional width $\mathscr{S}\left(1+l_{2} / l_{1}\right)^{1 / 2}$ and the vorticity falls by a factor of $\tilde{Y}_{0}^{2} / \mathscr{S}^{2}\left(1+l_{2} / l_{1}\right)$ (from (4.3)), whereas for a wide wake, the wake width and vorticity magnitude are hardly changed, except for diffusion which causes the width to permanently increase slightly, and the vorticity to fall.

During subsequent sequences of straining, the width of the wake at the start of the positive strained region is much larger than the straining-viscous lengthscale, regardless of the initial wake width $\left(Y_{0}\right)$. For an initially narrow wake, its subsequent dynamics are described, to leading order (for $n>1$ ), by

$$
\begin{gathered}
\tilde{Y}_{2 n+1}=\left(1+n\left(1+l_{2} / l_{1}\right)\right)^{1 / 2}, \quad \tilde{Y}_{2 n}=\left(n \mathscr{S}^{2}\left(1+l_{2} / l_{1}\right)\right)^{1 / 2}, \\
\frac{\Omega_{n}}{\Omega_{0}}=\frac{\tilde{Y}_{0}^{2}}{\frac{1}{2} n \mathscr{S}^{2}\left(1+l_{2} / l_{1}\right)} .
\end{gathered}
$$

Thus, the vorticity magnitude decreases substantially during the first positively strained region, whereas the decrease in the vorticity is thereafter negligible. For 

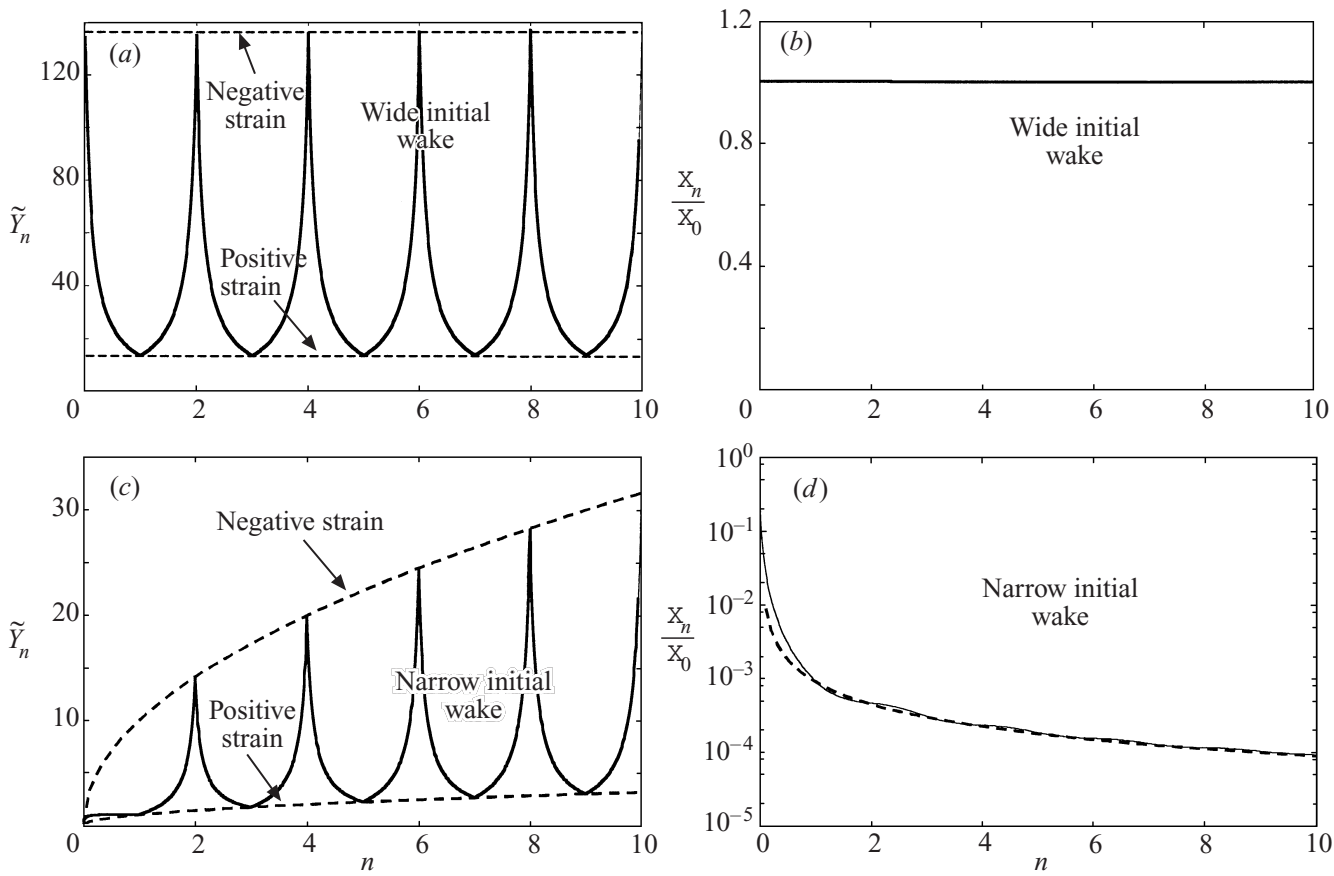

FIGURE 3. Comparison between similarity solutions and asymptotic expressions (4.4), (4.5) and (4.6) for the development of a wake subject to alternate positive and negative straining, characterized by $U_{1} / U_{0}=\mathscr{S}=10$ and $l_{1} / l_{2}=1$. The development of an initially wide wake, characterized by $\tilde{Y}_{0}=963$ is shown in $(a)$ and $(b)$, while an initially thin wake, characterized by $\tilde{Y}_{0}=0.3$, is shown in $(c)$ and $(d)$. The full curves correspond to calculations of the wake development using similarity solutions calculated in $\S 2.1$, while the dashed curves correspond to asymptotic expressions (4.4), (4.5) and (4.6).

the case of an initially wide wake, $(n>1)$,

$$
\tilde{Y}_{2 n+1}=\frac{\tilde{Y}_{0}}{\mathscr{S}}, \quad \tilde{Y}_{2 n}=\tilde{Y}_{0}, \quad \frac{\Omega_{n}}{\Omega_{0}}=1 .
$$

Figure 3 shows the development of a wide $\left(\tilde{Y}_{0} \gg \mathscr{S}\right)$ and a narrow wake $\left(\tilde{Y}_{0} \ll \mathscr{S}\right)$, respectively. In both cases, the strain parameter is large $(\mathscr{S}=10)$ and the length of the postively and negatively straining regions are chosen to be the same $\left(l_{2} / l_{1}=1\right)$. For comparison, the asymptotic expressions for the wake widths and vorticity magnitude (4.4), (4.5) and (4.6) are plotted and show good agreement with exact calculations using the similarity solutions derived in $\S 2.1$. Although the volume flux in the wake is conserved after alternate positive and negative straining $\left(Q_{2 n} / Q_{0}=1\right)$, it is extremely small in the positively strained regions where $Q_{2 n+1} / Q_{0}=1 / \mathscr{S}^{2}$.

These results show the net effect of alternate uniform straining on the development of a wake. The maximum velocity deficit in the wake, averaged over a positive and negative straining region, which we define as $\left\langle U_{w}\right\rangle=\left(l_{1} Y_{2 n+1} \Omega_{2 n+1}+l_{2} Y_{2 n} \Omega_{2 n}\right) /\left(l_{1}+l_{2}\right)$ is reduced slightly for the case of a wide wake, where $\left\langle U_{w}\right\rangle / U_{w 0} \sim l_{2} /\left(l_{1}+l_{2}\right)$, whereas for an initially narrow wake, the decrease is significant and is estimated to vary as $\left\langle U_{w}\right\rangle / U_{w 0} \sim l_{2} \tilde{Y}_{0} /\left(\left(l_{1}+l_{2}\right)^{3 / 2}(n \mathscr{S} / 2)^{1 / 2} \ll 1\right.$. Moreover, although the volume flux in the wake is recovered after a combination of positive and negative straining, the average volume flux over the intervals is small owing to the action of the positive strain, where $\langle Q\rangle / Q_{0} \sim 1 / \mathscr{S}^{2} \ll 1$. 

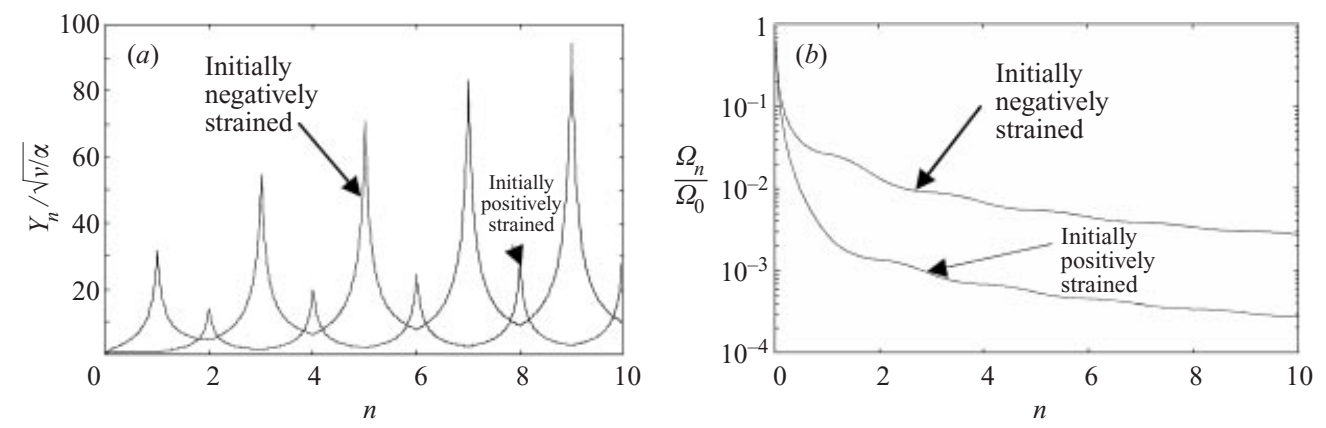

FIGURE 4. Demonstration of the non-ergodic nature of the straining field, where the straining rate is either initially positive or negative, and the initial width and strength of the wake is the same. The ratio between the maximum and minimum flow speed which is straining the flow is 9 and the length of the straining sections are chosen to be $l_{1} / l_{2}=1$. In $(a)$ the wake width is shown as a function of distance. The width is normalized by $\sqrt{v / \alpha}$ where $\alpha>0$ is the positive straining rate associated with a sequence of alternate straining sections. In $(b)$ the vorticity magnitude is normalized by its initial value. The figure shows the significant influence of the initial straining field, particularly when the wake is initially narrow.

When the strain rate of the initial segment of the flow is negative (i.e. $\alpha_{1}<0$ ), the wake width initially grows significantly with distance and the volume flux increases. When $0<\mathscr{S} \ll 1$, the asymptotic expressions developed for the case of an initially wide wake, (4.7), are still applicable. For an initially narrow wake, (4.4) and (4.5) apply, but with the modification that the expressions for $\tilde{Y}_{2 n}$ and $\tilde{Y}_{2 n+1}$ are interchanged. These results show that the development of a wake is sensitive to the order in which the strain rates are applied to the flow, as indicated in figure 4, so that a random sequence of straining is a non-ergodic process.

\subsection{Vorticity cancellation due to wake interaction}

The effect of positive strain is to confine the wake, enhancing vorticity cancellation, leading to the disappearance of the wake and reduction in the volume flux. The effect of wake confinement, by bounding walls or through wake-wake interaction with neighbouring bodies, also leads to vorticity cancellation. The calculation is straightforward but the results are important. Consider the effect of a series of parallel planar laminar wakes, separated by a distance $h$ and advected by a mean speed $\boldsymbol{U}_{E}=\left(U_{0}, 0\right)$. According to (2.3), the vorticity development is described by

$$
U_{0} \frac{\partial \omega}{\partial x}=v \frac{\partial^{2} \omega}{\partial y^{2}}
$$

Because of the effect of the bounding walls, or by symmetry,

$$
\frac{\partial \omega}{\partial y}=0
$$

at $y= \pm \frac{1}{2} h$. The body is located on the centreline, $y=0$. Applying the method of separation of variables and using the flow symmetry about $y=0$, shows that the vorticity is

$$
\omega(x, y)=\sum_{n=1}^{\infty} a_{n} \sin \left(\frac{2 n \pi y}{h}\right) \exp \left(-\frac{4 n^{2} \pi^{2} x v}{h^{2} U_{0}}\right)
$$


The volume flux associated with the flow (4.9) is

$$
Q(x)=-2 \int_{0}^{h / 2} y \omega \mathrm{d} x=-\sum_{n=1}^{\infty} \frac{2 a_{n}(-1)^{n+1}}{n \pi} \exp \left(-\frac{4 n^{2} \pi^{2} x v}{h^{2} U_{0}}\right) .
$$

Thus, the volume flux decays rapidly over a characteristic distance

$$
\frac{U_{0} h^{2}}{4 \pi^{2} v},
$$

corresponding to the time it takes for vorticity to diffuse laterally across a channel of width $h$.

When the bodies are arranged in a square lattice (e.g. Rollet-Miet et al. 1999), separated by a distance $h$, and the flow past them is characterized by a Reynolds number $R e_{b}=U_{0} D / v$, the downstream distance beyond which the flow is irrotational, expressed in relative size of the body separation is $O\left(\operatorname{Re}_{b}(h / 64 D)\right)$. Combining $\S \$ 4.1$ and 4.2 shows first that complex straining typically leads to an effective widening of wakes and enhanced decay of vorticity, and secondly, where wakes diffuse into one another, the vorticity decays quite quickly over a distance of order $\operatorname{Re}_{b}(h / 64 D)$.

\section{Concluding remarks}

In this paper, we have examined the widespread phenomenon of wake disappearance, which occurs when the wake is confined, due to the action of a positive straining field, or to wakes diffusing into one another or through the effect of walls. The same processes occur in both laminar and turbulent flows. We have also examined the effects of a sequence of positive and negative large-scale strains, and shown that these broaden the wake greatly but do not necessarily reduce the peak velocity or momentum flux in the wake. Their average effect is not like that of extra eddy diffusion; indeed, there may be a transfer of momentum flux to the wakes from the straining flows.

With constant positive strain, the volume flux in the wake decreases rapidly with downstream distance until the flow ultimately becomes irrotational, and the far-field flow is dipolar. The strength of the dipolar component is a combination of that resulting from the body in an unstrained flow, and a second new contribution due to the straining field. In a positively strained flow, the drag force on the body is now no longer proportional to the volume flux far downstream (which eventually tends to zero), but is determined by the momentum flux arising from the dipolar flow field. The total drag is essentially the sum of the viscous drag in uniform flows and the inviscid force arising from the straining flow. This analysis shows why viscous and inviscid forces may be superposed for moderate rates of external strain, which is consistent with the flow computations of Magnaudet, Rivero \& Fabre (1995) and two-phase flow models used in practice.

Even in unstrained flows, the wakes of bodies experience some straining caused by the flow over the body in the near field. This effect may be so large (if $\tilde{Y} \ll 1$ ) as in the case of bubbles and localized impulsive motions such as vortex rings, that the momentum flux of the downstream wake $\rho Q(0) U_{0}$ is much less than the drag on the object. In that case, Betz's formula for the drag is invalid and the drag is determined by laminar (or turbulent stresses) induced by the external flow, and by any entrainment processes across the control surface.

Disappearance and rapid annihilation of vorticity occurs in a number of different environmental flows. Isolated monopolar vorticities in the ocean which are char- 
acterized by an intense vortical core and surrounded by opposite signed vorticity (Kloosterziel 1990), decay rapidly owing to the diffusing of the inner core into the outer ring of opposite signed vorticity. When eddies are moving relative to the surrounding fluid, such as those in turbulent flows, they shed vorticity (e.g. Turner 1973; Rottman, Simpson \& Stansby 1987) and the mechanism of vorticity cancellation described in this paper means that the influence of the wake of the eddies or coherent structures downstream are generally negligible, so that they may be analyzed locally (except for their effect on the mean flow) and their downwind influences can be neglected.

The results in this paper, when applied to flow through groups of bluff bodies and eddies, indicate that the wakes tend to disappear because of vorticity cancellation due to the effect of strain and wake-wake interaction, but which process dominates depends on the pattern of the obstacles (or eddies). For staggered patterns of obstacles, the wakes' disappearance is determined by vorticity cancellation, whereas for in-line patterns, it is determined more by wake-wake interaction. For a random distribution, the distribution of the first rows may have a permanent effect, because the flows are not ergodic. The results, which need to be extended to the case where finite strains are applied over a limited extent of the wake, give a theoretical foundation for the assumption that the force on each object in relation to the local flow may be only weakly affected by wakes of upwind objects. It is important to note, however, that the entire flow field is affected by forces on all objects, and their combined effect may be estimated in terms of the distributed force exerted by collections of objects on the flow (Jerram, Belcher \& Hunt 1997).

I.E. gratefully acknowledges the support of EPSRC at University College London. These calculations arose from a wider study of the forces on particles in complex flow at the Department of Mechanical Engineering, Delft University of Technology which was supported by FOM. We are grateful for interesting conversations with Professor F. T. M. Nieuwstadt and Patrizia Bernardini at Delft University of Technology. J.C.R.H. also acknowledges the Natural Environment Research Council for its support of studies of complex flows.

\section{Appendix A}

The equivalence between the similarity solution and an exact solution to (2.3) is demonstrated here. The vorticity distribution downstream of a rigid body is assumed, say, to be of the form,

$$
\frac{\omega(y)}{\Omega(0)}=-\frac{y}{\left(\frac{1}{2} \pi\right)^{1 / 2} Y(0)} \exp \left(-\frac{y^{2}}{2 Y(0)^{2}}\right)
$$

which is located at the origin $\phi_{0}=0$ and the initial size of the wake is assumed to be much smaller than $(v /|\alpha|)^{1 / 2}$, that is $Y(0) \ll(v /|\alpha|)^{1 / 2}$. At the origin, $\psi^{\prime}=U_{0} y$. The downstream vorticity is thus

$$
\frac{\omega}{\Omega(0)}=-\int_{-\infty}^{\infty} \frac{\frac{y}{Y(0)} \exp \left(-\frac{y^{2}}{2 Y(0)^{2}}-\frac{\left(U_{0} y-\psi\right)^{2}}{4 v \phi}\right)}{\left(\frac{1}{2} \pi\right)^{1 / 2} \sqrt{4 \pi \phi v}} U_{0} \mathrm{~d} y .
$$


The above equation may be integrated exactly to give

$$
\frac{\omega}{\Omega(0)}=-\frac{U_{0}^{2} \psi}{\left(\frac{1}{2} \pi\right)^{1 / 2} Y(0)\left(U_{0}^{2}+2 v \phi / Y(0)^{2}\right)^{3 / 2}} \exp \left(-\frac{\psi^{2}}{4 v \phi}+\frac{\psi^{2} U_{0}^{2}}{4 v \phi\left(U_{0}^{2}+2 v \phi / Y(0)^{2}\right)}\right) .
$$

When $\alpha>0$, the streamfunction $\psi \rightarrow \alpha x y$ while the velocity potential $\phi \rightarrow \frac{1}{2} \alpha x^{2}$, in which case

$$
\frac{\omega}{\Omega(0)} \rightarrow-\frac{Y(0)^{2} \alpha}{v} \frac{y}{\left(\frac{1}{2} \pi\right)^{1 / 2}(v / \alpha)^{1 / 2}} \frac{1}{\left(\alpha x / U_{0}\right)^{2}} \exp \left(-\frac{y^{2}}{2 v / \alpha}\right),
$$

which is identical to the similarity solution (2.18) and (2.21) in the limit $\alpha x / U_{0} \gg 1$. When $\alpha<0$, the downstream vorticity tends to

$$
\frac{\omega}{\Omega(0)} \rightarrow-\frac{y}{\left(\frac{1}{2} \pi\right)^{1 / 2} Y(0) /\left(1+\alpha x / U_{0}\right)} \exp \left(-\frac{y^{2}}{2 Y(0)^{2} /\left(1+\alpha x / U_{0}\right)^{2}}\right),
$$

thus the width of the wake spreads as $Y(0) /\left(1+\alpha x / U_{0}\right)$ while the peak vorticity tends to a constant value, consistent with (2.24).

\section{Appendix B}

The volume flux associated with the laminar wake of a flat plate is calculated from integral constraints placed on the flow. The volume flux (associated with the wake) is a function of downwind distance and is denoted by $Q_{w}(x)$, where $x$ is the distance from the RSP. The volume flux ultimately tends to the value $Q(0)$ which denotes the volume flux far downwind of the body.

The laminar flow $(u, v)$ past a flat plate is described, to leading order, by

$$
\frac{\partial u^{2}}{\partial x}+\frac{\partial u v}{\partial y}=v \frac{\partial^{2} u}{\partial y^{2}}
$$

where gradients of pressure parallel to the plate are negligible because the streamlines are parallel. Writing

$$
\mathscr{M}(x)=\int_{-\infty}^{\infty}\left(u^{2}-U_{0}^{2}\right) \mathrm{d} y
$$

the momentum equation (B 1) may be expressed as

$$
\frac{\mathrm{d} \mathscr{M}}{\mathrm{d} x}=-\left[u v+v \frac{\partial u}{\partial y}\right]_{y=0-}^{0+}
$$

On the centreline, there is no vertical flow and $v=0$. When the vertical surface, over which the integration over $y$ takes place, does not cut the plate, the right-hand side of (B 2) is zero because the shear-free condition $\partial u / \partial y=0$ applies. However, when the vertical line over which the integration is taken, cuts the plate, the right-hand side of (B 2) is non-zero owing to the shear stress adjacent to the plate surface. From (B 2), we find that downwind of the plate $(x>0), \mathscr{M}$ is constant so that

$$
\mathscr{M}(x)=-\int_{-\infty}^{\infty} 2 U\left(U_{0}-u\right) \mathrm{d} y+\int_{-\infty}^{\infty}\left(u-U_{0}\right)^{2} \mathrm{~d} y=C,
$$

where $C$ is a constant. The first term on the right-hand side is linear in velocity, and is equal to the sum of the positive volume flux associated with the wake $\left(Q_{w}(x)\right)$ and 
the associated volume flux associated with a source term $\left(q_{s}(x)\right)$, so that

$$
Q_{w}(x)-q_{s}(x)=\frac{1}{2 U_{0}} \int_{-\infty}^{\infty}\left(u-U_{0}\right)^{2} \mathrm{~d} y+C .
$$

Downwind of the RSP, the volume flux in the wake and the source are related (by the conservation of the mass) through

$$
q_{s}(x)=\frac{1}{2} Q_{w}(x),
$$

where the factor of $\frac{1}{2}$ arises because the source also generates an upstream volume flux $q_{s}$. Far downwind, the right-hand side of (B 3) tends to a constant value $C$, because the square of the velocity deficit tends to zero faster than the wake spreads, while the left-hand side tends to $\frac{1}{2} Q(0)$. Thus, the volume flux in the wake is

$$
Q_{w}=Q(0)+\frac{1}{U_{0}} \int_{-\infty}^{\infty}\left(u-U_{0}\right)^{2} \mathrm{~d} y,
$$

which decreases monotonically with downstream distance.

\section{REFERENCES}

Arfken, G. 1985 Mathematical Methods for Physicists, 3rd edn. Academic.

Batchelor, G. K. 1967 An Introduction to Fluid Mechanics. Cambridge University Press.

BETZ, A. 1925 A method for the direct determination of profile drag. Z. Flugtechn. Motorluftschiffahrt. 16, 42 (in German).

Bowles, R. G. A. \& Smith, F. T. 2000 Lifting multi-blade flows with interaction. J. Fluid Mech. 400, $1-24$.

BoussinesQ, M. J. 1905 Sur le pouvoir refroidissant d'un courant liquide ou gazeux. J. de Math. 1, 285-290.

Couet, B., Brown, P. \& Hunt, A. 1991 Two-phase bubbly-droplet flow through a contraction: experiments and a unified model. Intl J. Multiphase Flow 17, 291-307.

Elliot, C. J. \& TownSEnd, A. A. 1981 The development of a turbulent wake in a distorting duct. J. Fluid Mech. 113, 433-467.

GrahaM, J. M. R. 1980 The forces on sharp-edged cylinders in oscillatory flow at low KeuleganCarpenter numbers. J. Fluid Mech. 97, 331-346.

Hunt, J. C. R. \& Mulhearn, P. J. 1973 Scalar dispersion in the vicinity of a two-dimensional body. J. Fluid Mech. 61, 245-274.

Hunt, J. C. R., Perkins, R. \& Fung, J. 1994 Problems in modelling disperse two-phase flows. Appl. Mech. Rev. 47, 49-60.

Jerram, N., Belcher, S. E. \& Hunt, J. C. R. 1997 Turbulent flow through a distributed force a model for the wind within and above an urban canopy. Proc. IMA Conf. Turb. Flow (ed. R. J. Perkins \& S. E. Belcher), pp. 157-173. Oxford University Press.

KefFer, J. F. 1965 The uniform distortion of a turbulent wake. J. Fluid Mech. 22, 135-159.

Keffer, J. F., Kawall, J. G., Hunt, J. C. R. \& Maxey, M. R. 1978 The uniform distortion of thermal and velocity mixing layers. J. Fluid Mech. 86, 465-490.

Kloosterziel, R. C. 1990 Barotropic vortices in a rotating fluid. PhD thesis. Utrecht University, The Netherlands.

Kowe, R., Hunt, J. C. R., Hunt, A., Couet, B. \& Bradbury, L. J. S. 1988 The effects of bubbles on the volume fluxes and the pressure gradients in unsteady and non-uniform flow of liquids. Intl J. Multiphase Flow 14, 587-606.

Magnaudet, J. \& Eames, I. 2000 Motion of high Re bubbles in inhomogeneous flow. Annu. Rev. Fluid Mech. 32, 659-708.

Magnaudet, J., Rivero, M. \& Fabre, J. 1995 Accelerated flows past a rigid sphere or a spherical bubble. Part 1. Steady straining flow. J. Fluid Mech. 284, 97-135.

Moore, D. W. 1963 The boundary layer on a spherical gas bubble. J. Fluid Mech. 16, 161-176. 
Neish, A. \& Smith, F. T. 1988 The turbulent boundary layer and wake of an aligned flat plate. J. Engng Maths 22, 15-42.

Parkinson, G. V. \& Jandali, T. 1970 A wake source model for bluff body potential flow. J. Fluid Mech. 40, 577-594.

Rollet-Miet, P., Laurence, D. \& Ferziger, J. 1999 LES and RANS of turbulent flow in tube bundles. Intl J. Heat Fluid Flow, 22, 241-254.

Rottman, J. W., Simpson, J. E. \& Stansby, P. K. 1987 The motion of a cylinder of fluid released from rest in a cross-flow. J. Fluid Mech. 177, 307-337.

Talke, F. E. \& Berger, S. A. 1970 The flat plate trailing edge problem. J. Fluid Mech. 40, 161-189. Turner, J. S. 1973 Buoyancy Effects in Fluids. Cambridge University Press. 